\title{
Identifying assessor differences in weighting the underlying sensory dimensions
}

\author{
El Mostafa QAnNARI ${ }^{(1)}$ Michael MeYNERS ${ }^{(2)}$ \\ ${ }^{(1)}$ ENITIAA/INRA - Unité de Statistique Appliquée à la Caractérisation des Aliments \\ Rue de la Géraudière B. P. 82 225, 44322 NANTES cedex 03 - FRANCE \\ ${ }^{(2)}$ Fachbereich Statistik, University of Dortmund, D-44221 DORTMUND, GERMANY.
}

\begin{abstract}
In a previous paper Kunert and Qannari (1999) discussed a simple alternative to Generalized Procrustes Analysis to analyze data derived from a sensory profiling study. After simple pre-treatments of the individual data matrices, they propose to merge the data sets together and undergo Principal Components Analysis of the matrix thus formed. On the basis of two data sets, it was shown that the results slightly differ from those obtained by means of Generalized Procrustes Analysis.

In this paper we give a mathematical justification to this approach by relating it to a statistical regression model. Furthermore, we obtain additional information from this method concerning the dimensions used by the assessors as well as the contribution of each assessor to the determination of these dimensions. This information may be useful to characterize the performance of the assessors and single out those assessors who downweight or overweight some dimensions. In particular, those assessors who overweight the last dimensions should arouse suspicion regarding their performance as, in general, the last dimensions in a principal components analysis are deemed to reflect random fluctuations.
\end{abstract}




\section{Introduction}

Kunert and Qannari (1999) propose a simple method for the analysis of sensory profiling data. They suggest simple pre-treatment of the individual data matrices to account for differences in scoring, such as shifts and use of different ranges of the scale. In a second step, the data matrices are merged together and a Principal Components Analysis (PCA) is performed on the supermatrix which includes the attributes of all assessors. On the basis of two data sets it was shown that the results of the proposed method match up to a large extent with those of Generalized Procrustes Analysis (GPA) (Gower, 1975; Arnold and Williams, 1986). Note that the method proposed by Kunert and Qannari is applicable to data obtained by means of a fixed vocabulary, i. e. all assessors use the same attributes, as well as to data derived from a free choice profiling procedure which allows each panelist to use his or her own set of attributes.

In this paper, we relate this approach to a linear regression model which states that there exist underlying sensory dimensions (latent variables) and that the observed attributes can be expressed as linear combinations of these dimensions. Originally this model was introduced by Tucker (1966) as a three-way factor analysis technique and was discussed within a sensory analysis framework by Brockhoff et al. (1996). This three-way factor analysis can be seen as a generalization of PCA and is therefore used to analyze differences between products, assessors and attributes simultaneously. We show useful properties of the method that enable a panel leader to identify differences between the assessors regarding their use of dimensions.

This paper can also be seen as an extension to the case of several data sets of a relationship between regression analysis and PCA exhibited by Jong and Kotz (1999). These authors give a link between these two statistical methods within the usual framework (two way data set). In sensory profiling analysis, we have several data sets that are similar in the sense that the panelists assess the same set of products.

The model has two interesting properties. On the one hand, it provides a sound statistical background and a strong justification to the method proposed by Kunert and Qannari (1999). On the other hand, it allows the panel leader to identify individual differences between the panelists with respect to both the number of dimensions used and the importance attached to the various underlying dimensions. Therefore, this paper can be seen as a sequel to the paper written by Kunert and Qannari (1999) as we discuss useful extensions and introduce indices which provide the practitioner with useful information. 
A noteworthy feature of the method presented herein is that it does not involve heavy computations. Both the PCA and the performance coefficients, which constitute the core subject of the present paper, can be simply computed with standard statistical software.

\section{Centering and pre-scaling the data sets}

The sensory profiling (free choice profiling or fixed vocabulary) of $n$ products by $m$ assessors results in $m$ matrices $X_{1}, X_{2}, \ldots X_{m}$, where the rows refer to the products and the columns to the attributes. These matrices are centered in order to remove the effects of judges scoring at different levels of the scale. The configurations $X_{1}, X_{2}, \ldots X_{m}$ are also pre-scaled in order to adjust for variations among assessors in range of scoring. For this purpose each configuration $X_{i}, i=1, \ldots$, mis multiplied by the isotropic scaling factor $\alpha_{i}$ given by :

$$
\alpha_{i}=\frac{\sqrt{T}}{\sqrt{t_{i}}},
$$

where ${ }_{i}$ is the sum of variances of the attributes in data matrix $X_{i}$ and

$$
T=\frac{\sum_{i=1}^{m} t_{i}}{m} .
$$

More details and a justification of these isotropic scaling factors are given by Kunert and Qannari (1999). In what follows, we consider the pre-scaled data sets denoted by $Y_{i}=\alpha_{i} X_{i}$ for $i=1, \ldots, m$.

\section{Determination of the underlying sensory dimensions}

The aim of the practitioner in sensory analysis is to depict relationships among products on the basis of a hopefully small number of dimensions (latent variables) that underlie the sensory perceptions expressed by the assessors. Let $C(n, q)$ denote the matrix which contains the $q$ underlying latent variables. These variables are assumed to be uncorrelated and to have variances equal to one. We assume that each assessor's attributes, which form the matrices $Y_{i}$ for $i=1, \ldots, m$, can be expressed as linear combinations of the latent variables given in $C$ plus some random errors. Formally, the model can be written as : 


$$
Y_{i}=C B_{i}+E_{i},
$$

where $B_{i}$ contains the coefficients of the linear combinations that link the attributes in $Y_{i}$ to the latent variables in $C$, and $E_{i}$ gives the random errors. This model relates to the well-known linear regression model. However, unlike multivariate regression analysis, we have to estimate several additional parameters : besides the estimation of $C$, we have to estimate also the matrices $B_{i}, i=1, \ldots, m$. In the context of sensory profiling, we are particularly interested in $C$ in order to describe the relationships among products. However, the different matrices $B_{i}$ provide useful information about the individual differences between the assessors with respect to the underlying dimensions.

\section{Estimation of the parameters}

We consider the well-known least squares criterion to estimate the parameters. This consists in minimizing the loss-function :

$$
\sum_{i=1}^{m} \operatorname{SSQ}\left(Y_{i}-C B_{i}\right)
$$

with respect to $C$ and $B_{i}$ for $i=1, \ldots, m$. In this expression, $\operatorname{SS} Q(A)$ denotes the sum of squares of all the elements for a given matrix $A$. In the following, the parameter estimates which are solutions to the minimization problem will also be denoted by $C$ and $B_{i}$, thus avoiding the cumbersome notations $\hat{C}$ and $\hat{B}_{i}$.

It can be shown that the $q$ columns of the estimated matrix $C$ are given by the first $q$ principal components of the matrix $Y=\left(Y_{1}\left|Y_{2}\right| \ldots \mid Y_{m}\right)$ which is formed by merging the individual data matrices $Y_{i}, i=1, \ldots, m$. Moreover, as it is well known from multivariate linear regression analysis, the matrices $B_{i}($ for $i=1, \ldots, m)$ are given by :

$$
B_{i}=\left(C^{T} C\right)^{-1} C^{T} Y_{i}
$$

where $C^{T}$ denotes the transpose of matrix $C$. Assuming that the columns of $C$ are uncorrelated and their respective variances being equal to one, we have:

$$
C^{T} C=n I,
$$


where I is the identity matrix. It follows that :

$$
B_{i}=\frac{1}{n} C^{T} Y_{i}
$$

Hence, the current element of matrix $B_{i}$ corresponding to the $(j, k)^{\text {th }}$ entry is the covariance between the $k^{\text {th }}$ attribute in matrix $Y_{i} \quad(\mathrm{i}=1,2, \ldots, \mathrm{m})$ and the $j^{\text {th }}$ standardized principal component of matrix $\mathrm{Y}$, i. e the $j^{\text {th }}$ column of matrix $C$.

The predicted individual assessor matrices are given by :

$$
\hat{Y}_{i}=C B_{i} .
$$

We can show that the loss function (3) can be written as :

$$
\sum_{i=1}^{m} \operatorname{SSQ}\left(Y_{i}\right)-\sum_{i=1}^{m} \operatorname{SSQ}\left(\hat{Y}_{i}\right)
$$

From the pre-scaling procedure, it follows that the quantity $\operatorname{SSQ}\left(Y_{i}\right)$ is a constant equal to $n T$ where $n$ is the number of products and $T$ is defined according to (2). Furthermore, $\operatorname{SS} Q\left(\hat{Y}_{i}\right)$ is the sum of squares explained by the model that consists in regressing $Y_{i}$ on $C$. The relationship

$$
\frac{1}{n} \sum_{i=1}^{m} \operatorname{SSQ}\left(\hat{Y}_{i}\right)=\lambda_{1}+\lambda_{2}+\cdots+\lambda_{q}
$$

links the latter term in (4) to the total variance explained by the $q$ principal components of $Y$. In this expression, $\lambda_{\mathrm{j}}(j=1, \ldots, q)$ are the eigenvalues associated with the principal components, i. e. the eigenvalues of $\frac{1}{n} Y Y^{T}$ or equivalent of $\frac{1}{n} Y^{T} Y$. Since the columns of $C$ are assumed to be uncorrelated, we can derive a more precise and more useful relationship by considering $\hat{Y}_{i}^{(j)}$, which is defined as the matrix obtained by regressing $Y_{i}$ on the $j^{\text {th }}$ principal component of $Y$ (i. e. the $j^{\text {th }}$ column of $C$ ). We have :

$$
\frac{1}{n} \sum_{i=1}^{m} \operatorname{SSQ}\left(\hat{Y}_{i}^{(j)}\right)=\lambda_{j},
$$

where $\operatorname{SSQ}\left(\hat{Y}_{i}^{(j)}\right)$ can be simply computed as the sum of squared covariances between the attributes in $Y_{i}$ and the $j^{\text {th }}$ principal component of $Y$ (i.e. $j^{\text {th }}$ column of matrix $C$ ).

We consider the index : 


$$
\lambda_{i j}=\frac{\operatorname{SSQ}\left(\hat{Y}_{i}^{(j)}\right)}{\operatorname{SSQ}\left(Y_{i}\right)}
$$

which reflects the percentage of total variance in $Y_{i}(i=1, \ldots, m)$ explained by the $j^{\text {th }}$ principal component of $Y$. It has been mentioned that $\operatorname{SS} Q\left(Y_{i}\right)=n T$, thus (6) simplifies to :

$$
\lambda_{i j}=\frac{S S Q\left(\hat{Y}_{i}^{(j)}\right)}{n T} .
$$

This index reflects the importance that assessor $i(i=1, \ldots, m)$ attaches to the $j^{\text {th }}$ dimension. If we consider all $q$ principal components associated with $Y$, it can be shown that

$$
\sum_{j=1}^{q} \lambda_{i j}=1
$$

for each assessor $i$. Roughly speaking, (8) expresses that each panelist provides us with an information regarding how the products differ from each others and the indices $\lambda_{i j}$ reflect how this information is partitioned into the various underlying dimensions.

Furthermore from (5) and (7) it also follows directly that :

$$
\frac{1}{m} \sum_{i=1}^{m} \lambda_{i j}=\frac{\lambda_{j}}{m T}
$$

Since for $i=1, \ldots, m$ the total variation in $Y_{i}$ is equal to $T$ owing two the prescaling procedure and therefore the total variation of $Y=\left(Y_{1}\left|Y_{2}\right| \ldots \mid Y_{m}\right)$ is equal to $m T$, the right side of equation (9) is precisely the percentage of total variance in $Y$ explained by the $j^{\text {th }}$ principal component of $Y$. Thus $\lambda_{i j}$ reflects the importance that assessor $i$ attaches to the $j^{\text {th }}$ dimension and the average over assessors reflects the relative importance of dimension $j$.

The various steps to calculate the underlying sensory dimensions and the performance indices are summarized in the appendix. 


\section{Examples}

Two examples will be given to illustrate the outcomes of the PCA on merged data sets (PCAMDS). We purposefully use the data that has already been analyzed by Kunert and Qannari (1999) and we do not depict relationships among products as this has already been discussed in their paper, but we focus on the assessor performances.

The first data set is obtained by means of a fixed vocabulary profiling procedure applied to 5 German beers. A panel of 13 assessors with no experience in sensory analysis participated in the test in which four attributes were considered (Kunert, 1998). The percentages of variation explained by the four underlying dimensions are $37.0 \%, 29.7 \%$, $19.9 \%$ and $13.5 \%$, respectively. Table 1 gives the indices $\lambda_{i j}$ from equation (7). They reflect the importance that the assessors attach to the underlying dimensions. The rather poor performance of the untrained panel is reflected by the high variation of the indices within each column. If we consider the first dimension for instance, we can see that the importance of this dimension is evaluated to $37.0 \%$ on average, which is exactly the percentage of variation explained by the first principal component (see the last row of table 1). However, this dimension explains only a small amount of variation in the configuration associated with assessor $2(7.0 \%)$, and contrariwise it turns out to be very important for assessors 3 and 7 (75.0\%). The same comments hold for the other dimensions with respect to other assessors. If we compare the rows in table 1, we can see that except for assessor 12 and, to a smaller extent, assessor 11, all assessors have one dominant dimension. For instance dimension 2 explains $79 \%$ of the total variation in the configuration associated with assessor 13 . Being untrained, these assessors seem to mainly assess the products according to only one latent variable. Considering assessor 12, the dimensions 1, 3 and 4 turn out to be equally important and the importance he or she gives to dimension 2 is also not negligible. Therefore, the configuration of assessor 12 is more or less spherical (equal importance of all the directions) which, considering the context, might be a hint that the assessor under consideration has just given random scores to the products. 
Table 1. Beer data: Percentage of variation in each assessor's configuration explained by the principal components.

\begin{tabular}{ccrrrr}
$\begin{array}{c}\text { dimension } \rightarrow \\
\text { assessor } \downarrow\end{array}$ & $\mathbf{1}$ & $\mathbf{2}$ & $\mathbf{3}$ & $\mathbf{4}$ & Total \\
\hline $\mathbf{A 1}$ & $15.0 \%$ & $15.0 \%$ & $17.0 \%$ & $53.0 \%$ & $100.0 \%$ \\
A2 & $7.0 \%$ & $66.0 \%$ & $23.0 \%$ & $4.0 \%$ & $100.0 \%$ \\
A3 & $75.0 \%$ & $14.0 \%$ & $10.0 \%$ & $1.0 \%$ & $100.0 \%$ \\
A4 & $55.0 \%$ & $3.0 \%$ & $14.0 \%$ & $27.0 \%$ & $100.0 \%$ \\
A5 & $15.0 \%$ & $49.0 \%$ & $19.0 \%$ & $17.0 \%$ & $100.0 \%$ \\
A6 & $11.0 \%$ & $30.0 \%$ & $58.0 \%$ & $1.0 \%$ & $100.0 \%$ \\
A7 & $75.0 \%$ & $11.0 \%$ & $9.0 \%$ & $4.0 \%$ & $100.0 \%$ \\
A8 & $65.0 \%$ & $4.0 \%$ & $14.0 \%$ & $16.0 \%$ & $100.0 \%$ \\
A9 & $61.0 \%$ & $26.0 \%$ & $10.0 \%$ & $3.0 \%$ & $100.0 \%$ \\
A10 & $21.0 \%$ & $49.0 \%$ & $22.0 \%$ & $8.0 \%$ & $100.0 \%$ \\
A11 & $41.0 \%$ & $24.0 \%$ & $30.0 \%$ & $5.0 \%$ & $100.0 \%$ \\
A12 & $28.0 \%$ & $14.0 \%$ & $24.0 \%$ & $33.0 \%$ & $100.0 \%$ \\
A13 & $11.0 \%$ & $79.0 \%$ & $9.0 \%$ & $2.0 \%$ & $100.0 \%$ \\
\hline average & $\mathbf{3 7 . 0 \%}$ & $\mathbf{2 9 . 7 \%}$ & $\mathbf{1 9 . 9 \%}$ & $\mathbf{1 3 . 5 \%}$ & $\mathbf{1 0 0 . 0 \%}$
\end{tabular}

The second data set was obtained from an experiment involving a free choice profiling procedure. The data are provided and discussed by Dijksterhuis and Punter (1990) and have also been analyzed by Dijksterhuis and Gower (1991) by means of GPA. In this experiment, seven assessors rated eight yogurts. Table 2 shows how much the assessors contribute to the different dimensions. There is less discrepancy between the importance attached by the assessors to each dimension than in the previous case. The indices associated with the assessors with respect to dimensions 1 and 2 are plotted in figure 1. It can be seen that assessors 2 and 5 bestow much importance upon dimension 1 whereas assessors 6 and 7 give less importance to this dimension. For assessor 7 we see that dimensions 1 and 2 together explain less than $50 \%$ of the total variation in the configuration. In table 2 it can be seen that this assessor gives even more importance to dimension 3 than to dimensions 1 and 2, respectively. 
Table 2. Yogurt data: Percentage of variation in each assessor's configuration explained by the principal components.

\begin{tabular}{ccccccccc}
$\begin{array}{c}\text { Dimension } \rightarrow \\
\text { Assessor } \downarrow\end{array}$ & $\mathbf{1}$ & $\mathbf{2}$ & $\mathbf{3}$ & $\mathbf{4}$ & $\mathbf{5}$ & $\mathbf{6}$ & $\mathbf{7}$ & Total \\
\hline $\mathbf{1}$ & $42.0 \%$ & $34.0 \%$ & $3.0 \%$ & $5.0 \%$ & $8.0 \%$ & $7.0 \%$ & $2.0 \%$ & $100.0 \%$ \\
$\mathbf{2}$ & $61.0 \%$ & $5.0 \%$ & $10.0 \%$ & $9.0 \%$ & $7.0 \%$ & $6.0 \%$ & $1.0 \%$ & $100.0 \%$ \\
$\mathbf{3}$ & $48.0 \%$ & $14.0 \%$ & $18.0 \%$ & $7.0 \%$ & $3.0 \%$ & $6.0 \%$ & $4.0 \%$ & $100.0 \%$ \\
$\mathbf{4}$ & $48.0 \%$ & $28.0 \%$ & $8.0 \%$ & $4.0 \%$ & $5.0 \%$ & $5.0 \%$ & $2.0 \%$ & $100.0 \%$ \\
$\mathbf{5}$ & $66.0 \%$ & $12.0 \%$ & $4.0 \%$ & $3.0 \%$ & $8.0 \%$ & $6.0 \%$ & $1.0 \%$ & $100.0 \%$ \\
$\mathbf{6}$ & $24.0 \%$ & $36.0 \%$ & $11.0 \%$ & $15.0 \%$ & $10.0 \%$ & $2.0 \%$ & $2.0 \%$ & $100.0 \%$ \\
$\mathbf{7}$ & $22.0 \%$ & $22.0 \%$ & $24.0 \%$ & $11.0 \%$ & $6.0 \%$ & $5.0 \%$ & $11.0 \%$ & $100.0 \%$ \\
\hline Average & $\mathbf{4 4 . 4 \%}$ & $\mathbf{2 1 . 6 \%}$ & $\mathbf{1 1 . 1} \%$ & $\mathbf{7 . 7} \%$ & $\mathbf{6 . 7 \%}$ & $\mathbf{5 . 3} \%$ & $\mathbf{3 . 3} \%$ & $\mathbf{1 0 0 . 0 \%}$
\end{tabular}

Figure 1. Percentage of variation in each assessor's configuration explained by the two first principal components; (*) refers to the average percentage of variation over assessors.

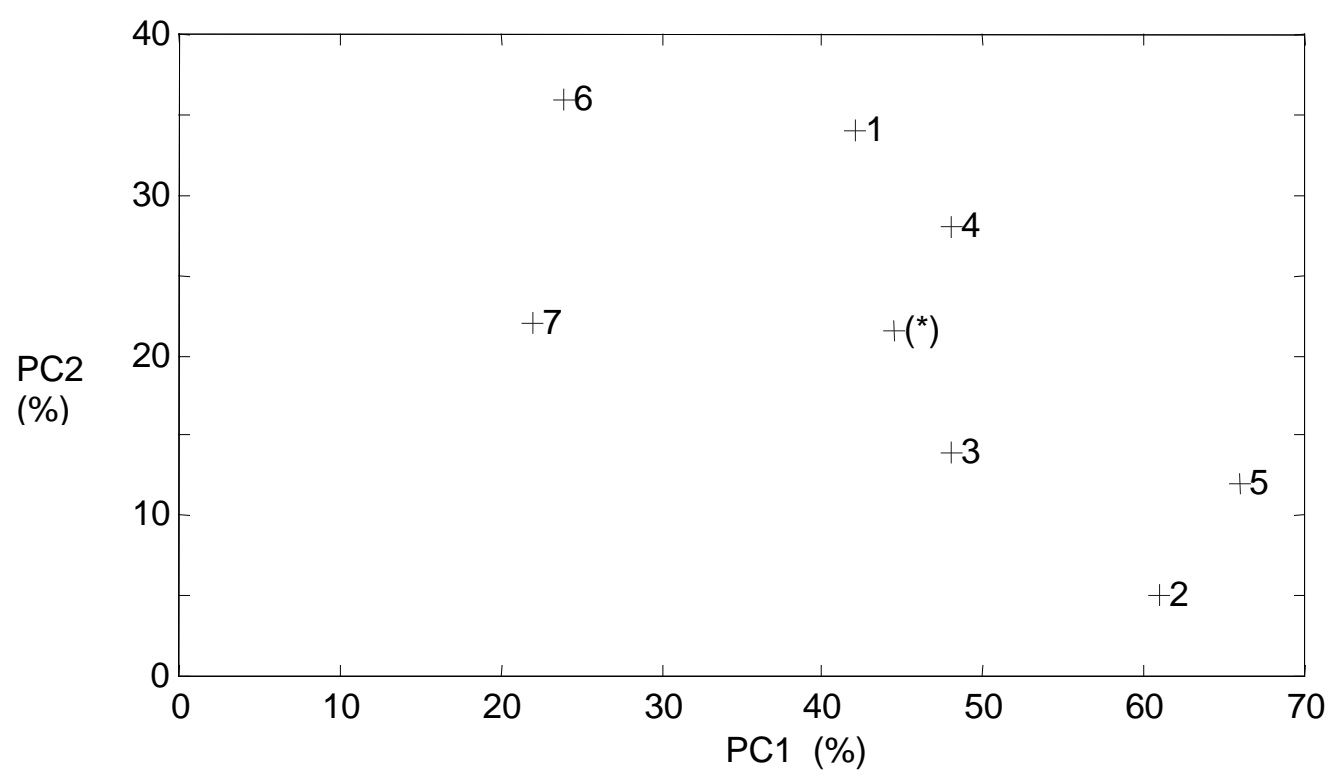




\section{Conclusion}

The paramount features of PCAMDS are, on the one hand, its simplicity and, on the other hand, its ability to provide performance indices associated with the assessors. These indices might be a simple tool for a panel leader to consider individual differences between the panelists with respect to their perception of different dimensions. Moreover, the method is supported by a statistical model that assumes the existence of latent variables which are related to the original data in the sense that the attributes can be retrieved as linear combinations of these underlying latent variables.

The method of analysis basically involves performing PCA on the merged data sets. Therefore, further developments of the method may rely on the wide statistical literature devoted to PCA which is by far the most popular method in multivariate analysis (Jolliffe, 1986). For instance, it is possible to extend the method of analysis to encompass the case where there are missing data (some assessors may be unavoidably retained from attending all sessions). Indeed, this is a tricky problem when using such method as GPA whereas solutions to this problem exist within PCA framework. This issue will be investigated in further research.

\section{Acknowledgement}

The second author is grateful to the Deutsche Forschungsgemeinschaft (SFB 475, "Reduction of complexity for multivariate data structures") for the financial support of this work. 


\section{References}

ARNOLD, G. M. and WILLIAMS, A. A. (1986). The use of Generalized Procrustes Analysis in sensory analysis. In: Statistical Procedures in Food Research, ed. J. R. Piggott. Elsevier. 233-253.

BROCKHOFF, P. M., HIRST, D. and NÆS, T. (1996). Analysing individual profiles by three way factor analysis. In: Multivariate analysis of data in sensory science, ed. T. Næs and E. Risvik. Elsevier. 307-342.

DIJKSTERHUIS, G. B. and GOWER, J. C. (1991). The interpretation of Generalized Procrustes Analysis and allied methods. Food Quality and Preference, 3, 67-87.

DIJKSTERHUIS, G. B. and PUNTER, P. H. (1990). Interpreting Generalized Procrustes Analysis 'analysis of variance' tables. Food Quality and Preference, 2, 255-265.

GOWER, J. C. (1975). Generalized Procrustes Analysis. Psychometrika, 40, 33-51.

JOLLIFFE, I. T. (1986). Principal Component Analysis. New York: Springer-Verlag.

JONG, J. C. and KOTZ, S. (1999). On a relation between Principal Component Analysis and regression analysis. The American Statistician, 53 (4), 349-351.

KUNERT, J. (1998). Sensory experiments as crossover studies. Food Quality and Preference, 9, 243-253.

KUNERT, J. and QANNARI, E. M. (1999). A simple alternative to Generalized Procrustes Analysis: Application to sensory profiling data. Journal of Sensory Studies, 14, 197208.

TUCKER, L. R. (1966). Some mathematical notes on three-mode factor analysis. Psychometrika, 31, 279-311. 


\section{Appendix}

The aim of this appendix is to summarize the various steps of the analysis.

Step 1 : Compute the isotropic scaling factors $\alpha_{i}(i=1, \ldots, m)$ according to (1) and set $Y_{i}=\alpha_{i} X_{i}$

Step 2 : Perform a PCA on the merged data tables $Y=\left(Y_{1}\left|Y_{2}\right| \ldots \mid Y_{m}\right)$; relationships among products can be depicted on the basis of the unstandardized principal components.

Step 3 : Denote by $c_{1}, c_{2}, \ldots c_{p}$ the standardized principal components associated with $Y$. For each data table $Y_{i}(i=1, \ldots, m)$ and for each principal component $c_{j}(j=1, \ldots, p)$ compute the percentage of total variance in $Y_{i}$ explained by $c_{j}$ according to

$$
\lambda_{i j}=\frac{S S Q\left(\hat{Y}_{i}^{(j)}\right)}{n T}
$$

where $\operatorname{SSQ}\left(\hat{Y}_{i}^{(j)}\right)$ is computed as the sum of the squared covariances between the attributes in $Y_{i}$ and $c_{j}, n$ is the number of products and $T$ is defined according to (2). $\lambda_{i j}$ reflects the importance that assessor $i$ attaches to dimension $c_{j}$. These indices can be compared to each other and to their average, which is as a matter of fact the percentage of variation explained by $c_{j}($ step 2$)$. 